TITLE:

\title{
Magnetic field stability control of HTS-MRI magnet by use of highly stabilized power supply
}

\section{$\operatorname{AUTHOR}(\mathrm{S})$ :}

Yachida, Takayuki; Yoshikawa, Masaki; Shirai, Yasuyuki; Matsuda, Tetsuya; Yokoyama, Shoichi

\section{CITATION:}

Yachida, Takayuki ... [et al]. Magnetic field stability control of HTS-MRI magnet by use of highly stabilized power supply. IEEE Transactions on Applied Superconductivity 2017, 27(4): 4702905.

ISSUE DATE:

2017-06

URL:

http://hdl.handle.net/2433/228377

\section{RIGHT:}

(c) 2017 IEEE. Personal use of this material is permitted. Permission from IEEE must be obtained for all other uses, in any current or future media, including reprinting/republishing this material for advertising or promotional purposes,

creating new collective works, for resale or redistribution to servers or lists, or reuse of any copyrighted component of this work in other works.; This is not the published version. Please cite only the published version.; この論文は出版社版

でありません。引用の際には出版社版をご確認ご利用ください。 


\title{
Magnetic Field Stability Control of HTS-MRI Magnet by Use of Highly Stabilized Power Supply
}

\author{
T. Yachida, M. Yoshikawa, Y. Shirai, Member IEEE, T. Matsuda and S. Yokoyama
}

\begin{abstract}
Low temperature superconducting (LTS)-MRI magnets are usually cooled by a large amount of liquid helium (LHe), however LHe is getting scarcer and its price has been rising. Therefore, development of helium free high temperature superconducting (HTS)-MRI magnet is expected.
\end{abstract}

HTS-MRI magnet fabricated by REBCO tape superconductors has mainly two problems for producing a highly stabilized magnetic field for MRI imaging. One problem is current fluctuation of a power supply, which is necessary for excitation because the permanent current operation of HTS-MRI magnet is difficult. Another is a screening current in REBCO tapes which affect the magnetic field stability.

The MRI magnetic field feedback control test was carried out, that is, the overshooting and the current feedback control, using 32H HTS-MRI test magnet and the highly stabilized power supply. The magnetic field was evaluated under the current feedback control at $1.5 \mathrm{~T}(66 \mathrm{~A})$. By the overshooting and the current feedback control, the magnetic field stability was improved to $0.7 \mathrm{ppm} / \mathrm{hr}$ which is enough for MRI imaging.

Index Terms - Magnetic resonance imaging, HTS magnets, Magnet stability, Electromagnet power supplies, Magnetization, Magnetic field measurement, Magnetic field quality.

\section{INTRODUCTION}

$\mathrm{T}$ HE low temperature superconducting (LTS) MRI magnets are widely and commercially applied worldwide. They are cooled by a large amount of LHe, however LHe is getting scarcer and its price has gone up. Therefore, It is expected to develop a conduction cooling HTS-MRI magnet.

The high temperature superconducting (HTS) MRI magnet fabricated by REBCO tape superconductors has mainly two problems for producing a very stable magnetic field for MRI imaging. One difficulty is that the superconducting junction of

Automatically generated dates of receipt and acceptance will be placed here; authors do not produce these dates. This work was supported by

"Development of Medical Devices and Systems for Advanced Medical Services" from Ministry of Economy, Trade and Industry (METI) and Japan Agency for Medical Research and Development (AMED). And a part of this paper is based on results obtained from a project commissioned by the New Energy and Industrial Technology Development Organization (NEDO).

T. Yachida and Y. Shirai are with Graduate School of Energy Science, Kyoto University, Kyoto 606-8501, Japan, e-mail: yachida@ @e.energy.kyotou.ac.jp; shirai.yasuyuki.7v@kyoto-u.ac.jp.

M. Yoshikawa was with Graduate School of Energy Science, Kyoto University, Kyoto 606-8501, Japan, e-mail: yoshikawa@pe.energy.kyotou.ac.jp.

T. Matsuda and S. Yokoyama are with Mitsubishi Electric Corporation, Amagasaki Hyogo 661-8661, Japan, e-mail: Matsuda.tetsuya@dn.mitsubishielectric.co.jp; $\quad$ Yokoyama.Shoichi@ dx.MitsubishiElectric.co.jp.
REBCO tapes with extremely low resistivity necessary for the persistent current operation of MRI magnet is hard to achieve by now. One solution is to use a power supply to keep a magnetic field constant even in MRI imaging operation instead of the persistent current operation. We must develop a highly stabilized power supply with precise current control to keep the MRI magnetic field constant.

Another important problem is long-lasting attenuation of screening currents induced on the tapes at the initial excitation of the HTS-MRI magnet. The screening current is induced by time-variation of flux linked to the tape surface. This attenuation affects the magnetic field stability both temporally and spatially with large time constant. As a solution to this problem, the overshooting operation is commonly used at the initial excitation (see Fig.5) [1]. The exciting current exceeds the target current once to a certain extent and then decreases to the target current in order to reduce the screening current. As for them, the electromagnetic behavior of HTS coils and the magnetic field stability were studied [2], [3].

With such a background, we have studied for improving the magnetic field of a HTS-MRI magnet using the highly stabilized power supply. A conduction cooled 3T HTS-MRI magnet $(32 \mathrm{H})$ was designed and fabricated (Fig.1). The cool down test, the excitation test (up to $3.0 \mathrm{~T}$ ) and the magnetic field distribution trimming test were successfully done [4].

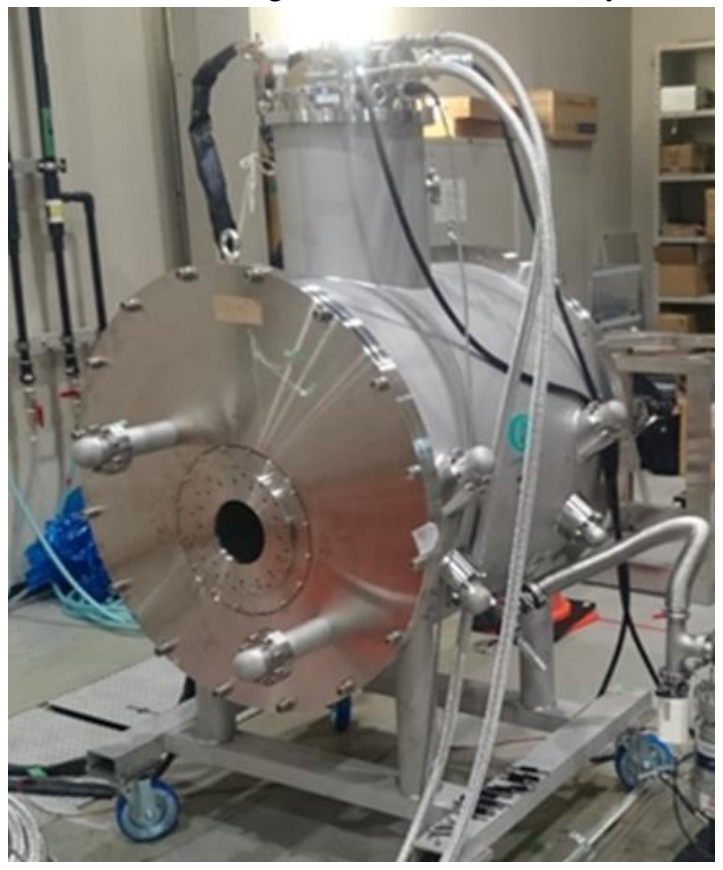

Fig. 1. 3T HTS-MRI magnet. 
Also, a highly stabilized excitation power supply was designed and tested (Fig.2). The clear MRI image was obtained under the magnetic field control of the power supply instead of the persistent current mode using a commercial LTS-MRI magnet [5].

In this paper, we focused on the highly-stabilized power supply equipped with a current feedback control which is newly developed in order to improve the magnetic stability of the HTS-MRI magnet. The target magnetic stability is less than $1.0 \mathrm{ppm} / \mathrm{hr}$ which is required for obtaining clear MRI image. The availability of the current feedback control was evaluated experimentally.

\section{EXPERIMENTAL APPARATUS}

\section{A. 3T HTS- MRI magnet}

The specification of the conduction cooled HTS-MRI magnet is shown in Table I. The magnet was composed of several pancake coils made by REBCO superconducting wires The specification of the REBCO wire is shown in Table II.

The magnetic field stabilization tests were carried out at $1.5 \mathrm{~T}(66 \mathrm{~A})$ which is almost half of the rated field of the magnet $(2.9 \mathrm{~T})$ so as to have some margin for flexibility of the test operation.

TABLE I

SPECIFICATION OF THE HTS-MRI MAGNET

\begin{tabular}{ll}
\hline \hline Magnetic field at center & $2.9 \mathrm{~T}$ \\
Coil inside diameter & $320 \mathrm{~mm}$ \\
Coil outside diameter & $471 \mathrm{~mm}$ \\
Coil axial length & $440 \mathrm{~mm}$ \\
Magnetic field uniformity (100mm sphere) & $1.67 \mathrm{ppm}$ \\
Rated current & $125 \mathrm{~A}$ \\
Inductance & $32.0 \mathrm{H}$ \\
Operating temperature & $10 \mathrm{~K}$ \\
Current density of the coil & $113 \mathrm{~A} / \mathrm{mm} 2$ \\
\hline \hline
\end{tabular}

TABLE II

SPECIFICATION OF REBCO WIRE USED FOR THE COIL

\begin{tabular}{lll}
\hline \hline Dimension & Conductor width & $5 \mathrm{~mm}$ \\
& Conductor thickness & $0.16 \mathrm{~mm}$ \\
Electrical & Critical current & $\begin{array}{l}\text { 250A or higher at 77K, } \\
\text { self-field }\end{array}$ \\
characteristics & & \\
\hline
\end{tabular}

\section{B. Power supply system for excitation and hold}

It is considered that the persistent current mode operation of the LTS-MRI magnet is by now difficult for the HTS-MRI magnet. The designed HTS-MRI magnet is always driven by the power supply, even in the imaging operation. The output current fluctuation of the power supply directly leads to the magnetic field fluctuation. Therefore the power supply used for current hold should be of a small capacity (low loss) but a highly stabilized. While the power supply used for excitation should be of a certain capacity for charge/discharge.

Then we introduced the highly stabilized power supply system for HTS-MRI magnet. The system composed of the excitation power supply, the current hold power supply, the protective resister, the quench detection unit and control unit. The specification of the power supply for excitation and for

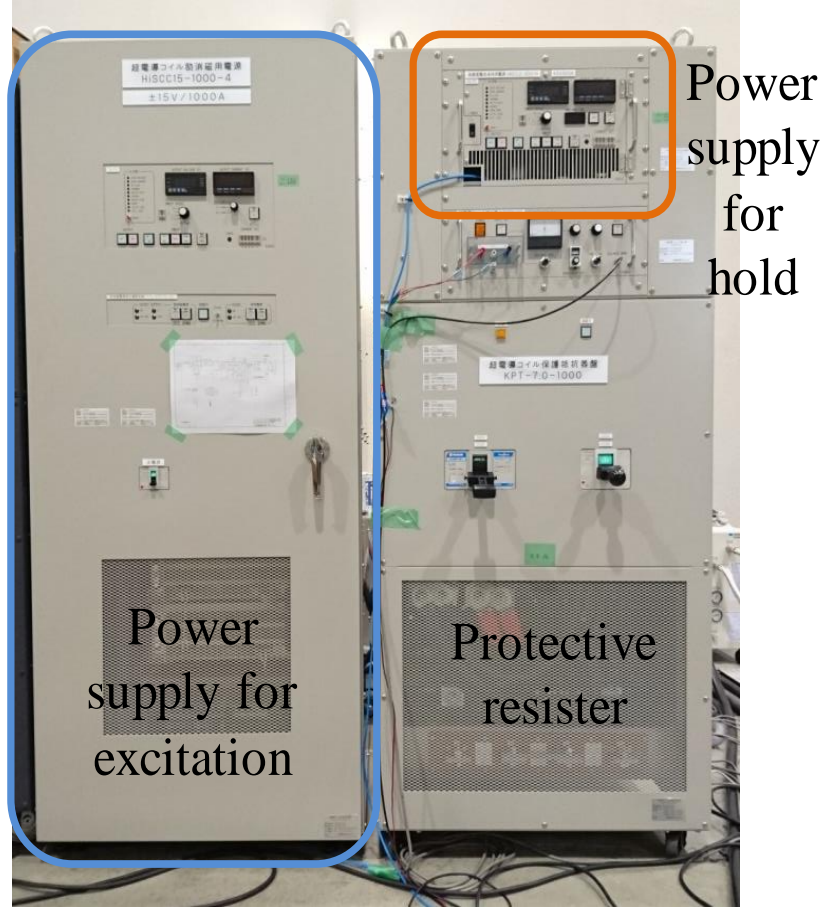

Fig. 2. Highly stabilized power supply for HTS-MRI magnet. Power supply for excitation is on the left and power supply for hold is on the upper right.

TABLE III

SPECIFICATION OF THE POWER SUPPLY FOR EXCITATION AND HOLD

\begin{tabular}{|c|c|c|}
\hline Item & Excitation & Hold \\
\hline Max output current & $1000 \mathrm{~A}$ & $300 \mathrm{~A}$ \\
\hline Output voltage & $\pm 15 \mathrm{~V}$ & $\pm 2 \mathrm{~V}$ \\
\hline Current stability & $100 \mathrm{ppm} / \mathrm{hr}$ & $1 \mathrm{ppm} / \mathrm{hr}$ \\
\hline $\begin{array}{l}\text { Output current ripple } \\
\text { (peak to peak) }\end{array}$ & 100ppm & $10 \mathrm{ppm}$ \\
\hline Cooling method & $\begin{array}{l}\text { Water cooling and } \\
\text { forced air cooling }\end{array}$ & Forced air cooling \\
\hline
\end{tabular}

hold is shown in Table III and the photograph is shown in Fig.2.

At the maintenance of the MRI magnet, it should be discharged, and after that, it should be charged again at an appropriate current sweep rate $\mathrm{d} I / \mathrm{d} t$ which is acceptable for the magnet.

While in the MRI imaging operation mode, the magnet current must be kept constant for the highly stable magnetic field with precise current control of the power supply. The power supply for excitation is only used in the maintenance mode and can be removed in the imaging mode. Instead of that, the power supply for hold with precise control of the magnetic field is used for MRI imaging operation.

So, total average efficiency of the power supply is improved with this proposed system compared with a single power supply system.

An important problem of the system to be solved is how to switch the connection from the magnet to the excitation power supply or to the current hold power supply with keeping the magnet current without any malfunction of the quench detection system, which is active throughout the MRI magnet operation. 


\section{MICRO CURRENT CONTROL EXPERIMENT}

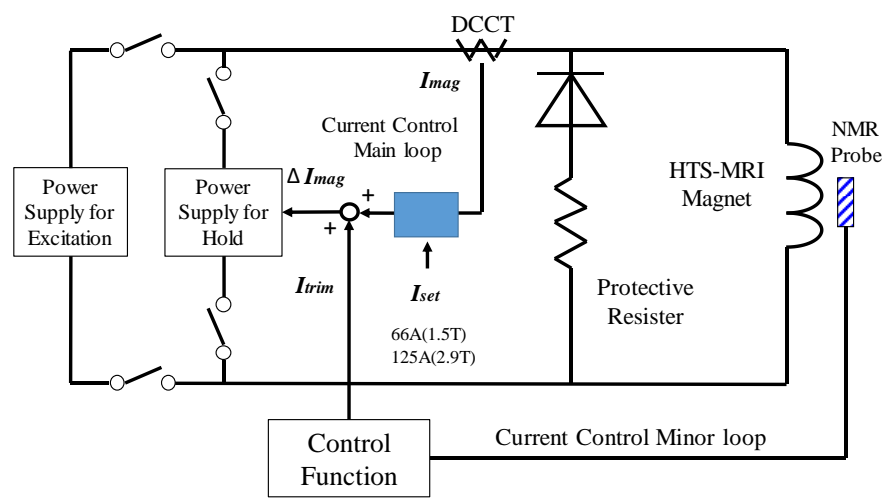

Fig. 3. Experimental circuit of micro current control (minor loop).

The switching control strategy between two power supply under exciting condition was designed and equipped in order not to generate a spike voltage at the switching, which may cause the misactivation of quench detection or actual quench of the magnet. The availability of the control strategy was confirmed experimentally.

\section{Micro current control function}

The magnet current (Imag) is kept almost constant at $66 \mathrm{~A}$ $\left(=I_{\text {set }}\right)$ by the current control main loop of the power supply for hold as shown in Fig.3. However, the necessary magnetic field stability for fine MRI imaging is less than $1 \mathrm{ppm}$ corresponding to the current resolution of $67 \mu \mathrm{A}$ at $1.5 \mathrm{~T}$, which is below the detection limit of DCCT.

Then, we designed a micro current control function (current control minor loop). The magnet current can be trimmed according to the control signal $\left(I_{\text {trim }}\right)$ in the range of $\mu \mathrm{A}$, which is given from the center magnetic field of MRI magnet measured by a NMR probe.

The micro current control parameter is shown in TABLE IV. In the experiments, the NMR probe measures the precise magnetic field (resolution $0.1 \mu \mathrm{T} ; 0.066 \mathrm{ppm}$ at $1.5 \mathrm{~T}$ ) every $5 \mathrm{~s}$. The control scheme is the followings (see Fig.4); if the measured field continuously exceeds the target field (=threshold value) during $15 \mathrm{~s}$ (threshold time), the trimming signal decreases $4.4 \mu \mathrm{A}(\sim 0.1 \mu \mathrm{T}$ at $1.5 \mathrm{~T})$. Conversely, it dips from the target during $15 \mathrm{~s}$, the signal increases $4.4 \mu \mathrm{A}$. The trimming output unit $(4.4 \mu \mathrm{A})$ is determined by minimum detection limit $0.1 \mu \mathrm{T}$ of the NMR probe.

TABLE IV

PARAMETER OF THE MiCRO CURRENT CONTROL

$\begin{array}{lc}\text { Target value } & 1.5358285 \mathrm{~T} \\ \text { Threshold value } & 1.5358285 \mathrm{~T} \\ \text { Threshold time } & 15 \mathrm{~s} \\ \text { Output increment } & 4.4 \mu \mathrm{A}(0.1 \mu \mathrm{T})\end{array}$

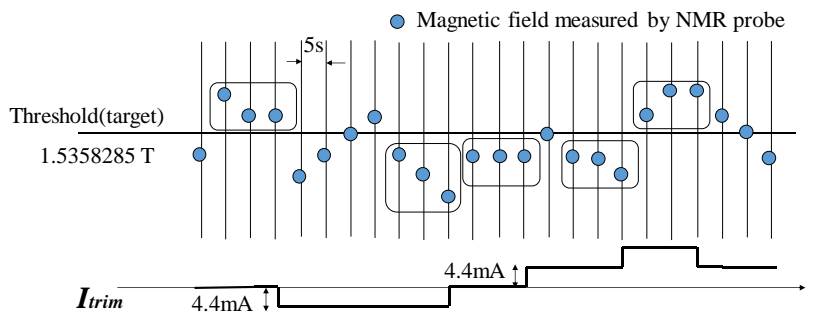

Fig. 4. Control scheme of the micro current control.
We excited the HTS-MRI magnet from 0A to 66A (1.5T) at $0.1 \mathrm{~A} / \mathrm{s}$ with $\Delta \mathrm{A}=0,4,8.12 \mathrm{~A}$ overshooting by the power supply for excitation. Outline of the overshooting is shown in Fig.5. $\Delta \mathrm{A}$ means overshoot current which exceeds the target current $(66+\Delta \mathrm{A} \mathrm{A})$.

After the overshooting, the power supply was switched to the power supply for hold, and the magnetic field stability measurement test was started for one hour with only main current control of the power supply.

Secondly, we excited again from 0A to 66A with the overshooting, switched to the power supply for hold, and then we started to add the minor control (micro current control) and measured the magnetic field stability for one hour for comparison.

\section{RESULTS AND DISCUSSION}

Fig.6 shows the magnetic field fluctuation for 15 minutes at $1.5 \mathrm{~T}$ (66A) without overshooting $(\Delta \mathrm{A}=0)$. That shows the magnetic field at the central position was increasing after starting the main constant current control. The deviation was $50 \mathrm{ppm}$ per 15 minutes and still increasing. It is estimated that the result is mainly due to the attenuation of the screening current of the superconducting tapes witch was induced in the charging operation. [1]

Fig.7 shows the magnetic field fluctuation for one hour at

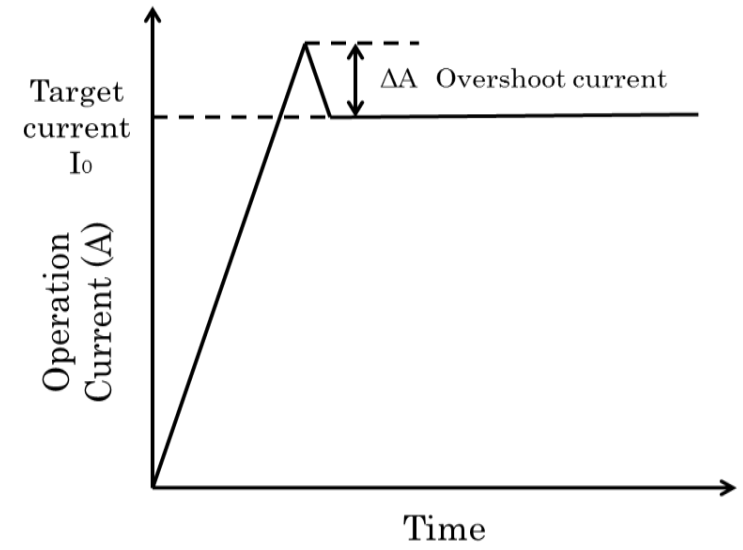

Fig. 5. Outline of the overshoot excitation.

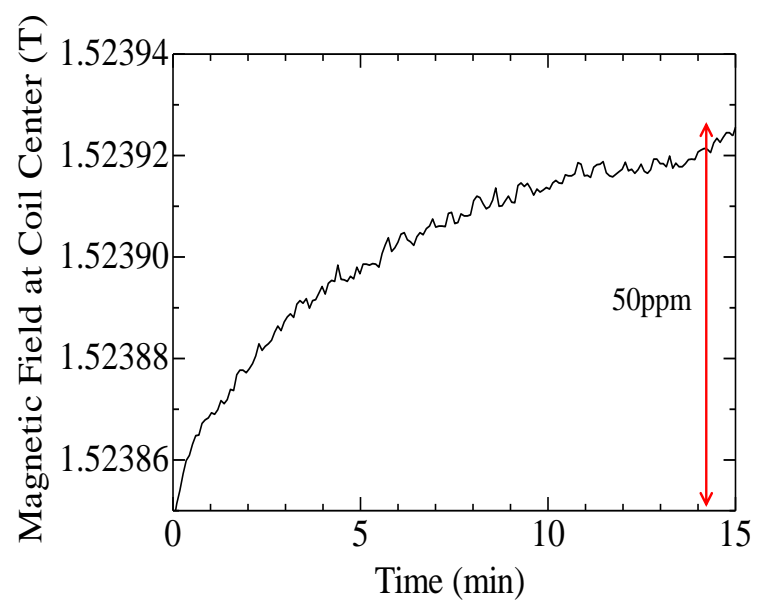

Fig. 6. The magnetic field fluctuation for 15 minutes at $1.5 \mathrm{~T}(66 \mathrm{~A})$ without overshoot excitation. 


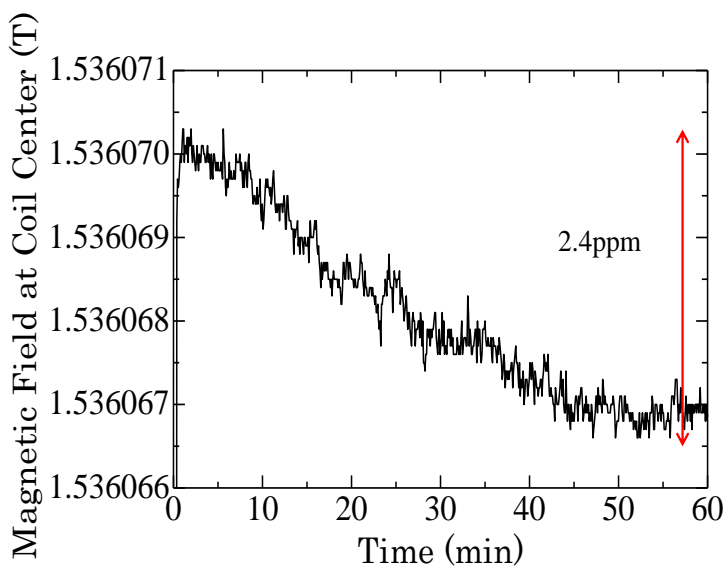

Fig. 7. The magnetic field stability for one hour at $1.5 \mathrm{~T}(66 \mathrm{~A})$ after $\Delta \mathrm{A}=8 \mathrm{~A}$ overshoot excitation with only main current control.

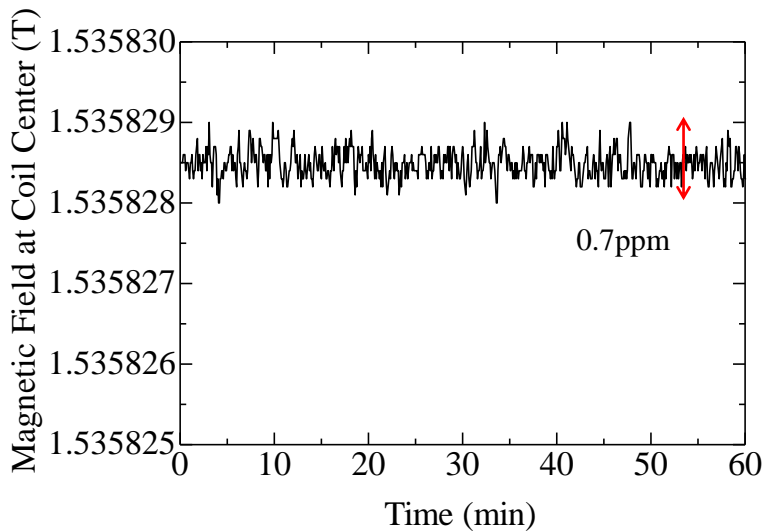

Fig. 8. The magnetic field stability for one hour at $1.5 \mathrm{~T}(66 \mathrm{~A})$ after $\Delta \mathrm{A}=8 \mathrm{~A}$ overshoot excitation with micro current control.

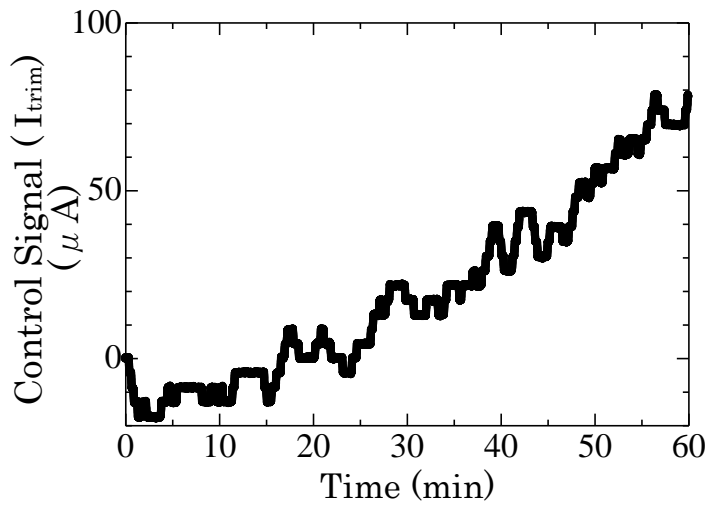

Fig.9. Control signal $\left(I_{\text {trim }}\right)$ of the micro current control function for power supply for hold.

$1.5 \mathrm{~T}\left(\mathrm{I}_{0}=66 \mathrm{~A}\right)$ after $\Delta \mathrm{A}=8 \mathrm{~A}$ overshooting with only main current control. The magnetic field fluctuation was considerably reduced to $2.4 \mathrm{ppm} / \mathrm{hr}$. If the overshoot current $\Delta \mathrm{A}$ can be still optimized, the field stability may be improved some more, but it is difficult to satisfy the stability level for imaging, $1 \mathrm{ppm} / \mathrm{hr}$ or less. The causes of the magnetic field fluctuation were considered as not only the attenuation of screening current but also the fluctuation of the power supply current control, however have not been clarified yet.

Fig. 8 shows the magnetic field stability for one hour at $1.5 \mathrm{~T}$ (66A) after $\Delta \mathrm{A}=8 \mathrm{~A}$ overshooting with the micro current control (minor control loop). The magnetic field was kept almost constant to the target field $(1.5358285 \mathrm{~T})$ with $0.7 \mathrm{ppm} / \mathrm{hr}$, which satisfies the fluctuation level of $1 \mathrm{ppm} / \mathrm{hr}$ or less required for MRI imaging.

Fig.9 shows the control signal $\left(I_{\text {trim }}\right)$ of the micro current control function for the power supply for hold. The control signal tends to increase and this shows the decrease of the magnetic field was suppressed by the micro current control.

Eventually we improved the magnetic field fluctuation from $50 \mathrm{ppm}$ per 15 minutes to $0.7 \mathrm{ppm} / \mathrm{hr}$ by the overshooting and the micro current control.

\section{CONCLUSION}

In developing the HTS-MRI magnet, it is one of the important issue to improve the magnetic field stability of the MRI magnet under the power supply driven mode. We have designed and made the 3T MRI test magnet with REBCO tape, and have performed excitation tests successfully. Also, the highly stabilized power supply system for excitation and for hold was proposed and fabricated. The system composed of the power supply for excitation, the power supply for hold, the protective resister, the quench detection unit and control unit. The former power supply is only for charging and discharging, and the latter one is for precise current control.

Using the test magnet, the magnetic field stability improvement test was carried out with the overshooting method and the micro current control by use of the proposed power supply system.

In conclusion, the magnetic field fluctuation $(50 \mathrm{ppm}$ per 15 minute) at the beginning of the constant current control was reduced to $2.4 \mathrm{ppm} / \mathrm{hr}$ by the overshooting.

In addition, the micro current control (the magnetic field feedback control), reduced the fluctuation to $0.7 \mathrm{ppm} / \mathrm{hr}$ which is sufficient for fine MRI imaging.

In this study, the magnetic field spatial uniformity was not considered. We will discuss the temporal trend of the field spatial distribution and the stability control strategy in the next step.

\section{REFERENCES}

[1] Y. Yanagisawa and H. Maeda, "Mechanism and Suppressive Methods for Screening-current-induced Magnetic Field of REBCO Coils", Cryogenics, Vol 48, No.4, pp. 165-171, 2013

[2] Y. Yanagisawa, et al, "Effect of current sweep reversal on the magnetic field stability for a Bi-2223 superconducting solenoid", Physica C, Vol. 469, pp. 1996-1999, 2009.

[3] T. Hemmi, et al. "Electromagnetic Behavior of HTS Coils in persistent current operations.", Fusion Engineering Design, 81, pp 2463-2466, 2006.

[4] Shoichi Yokoyama*, Jiwon Lee, Takeshi Imura, Tetsuya Matsuda, Tatsuya Inoue, Ryo Eguchi, Toshinari Ngahiro, Hajime Tanabe, Shinji Sato,Taketsune Nakamura, Yasuyuki Shirai, Daisuke Miyagi, Makoto Tsuda, "Research and Development of the Very Stable Magnetic Field HTS Coil System Fundamental Technology for MRI", SA-9-INV, ISS2015, Nov. 2015

[5] M. Yoshikawa, N. Yonemura, T. Yachida, T. Imura, Y. Shirai, S. Yokoyama, "Magnetic Field Stability test of LTS MRI Magnet Excited 
by Highly-stabilized Power Supply" , IEEE TRANSACTIONS ON APPLIED SUPERCONDUCTIVITY, VOL. 26, NO. 3, APRIL 2016, 4401105, Digital Object Identifier 10.1109/TASC.2016.2525958 